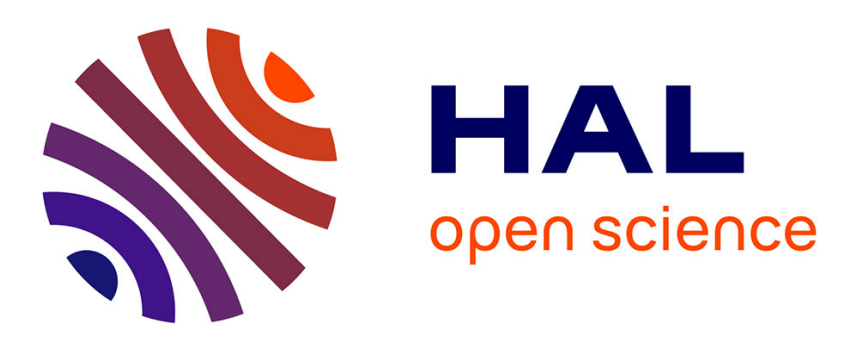

\title{
Essai d'évaluation de l'organisation et de la réorganisation de l'azote minéral du sol par la méthode des modèles compartimentaux I. - Cas d'un sol maintenu sans culture
}

Robert Morel, Thérèse Lasnier

\section{To cite this version:}

Robert Morel, Thérèse Lasnier. Essai d'évaluation de l'organisation et de la réorganisation de l'azote minéral du sol par la méthode des modèles compartimentaux I. - Cas d'un sol maintenu sans culture. Agronomie, 1981, 1 (1), pp.19-26. hal-00884218

\section{HAL Id: hal-00884218 https://hal.science/hal-00884218}

Submitted on 1 Jan 1981

HAL is a multi-disciplinary open access archive for the deposit and dissemination of scientific research documents, whether they are published or not. The documents may come from teaching and research institutions in France or abroad, or from public or private research centers.
L'archive ouverte pluridisciplinaire HAL, est destinée au dépôt et à la diffusion de documents scientifiques de niveau recherche, publiés ou non, émanant des établissements d'enseignement et de recherche français ou étrangers, des laboratoires publics ou privés. 


\title{
Essai d'évaluation de l'organisation et de la réorga- nisation de l'azote minéral du sol par la méthode des modèles compartimentaux I. - Cas d'un sol maintenu sans culture
}

\author{
Robert MOREL \\ avec la collaboration technique de Thérèse LASNIER
}

Chaire de Physico-Chimie et Science du Sol, Institut National Agronomique Paris-Grignon, 78850 Thiverval. Grignon.

RESUME

$N$ minéral du sol; Immobilisation ;

Organisation ; Minéralisation ; Sol nu,

Modèle compartimental.
Il est proposé dans ce mémoire un modèle compartimental établi d'après le critère de labilité (ou de stabiiité) de la matière organique du sol : un compartiment I comprend l'ensemble des substances organiques labiles, vivantes ou non vivantes; un compartiment II rassemble les substances organiques stabilisées, non vivantes et de faible réactivité ; ce dernier compartiment représente une masse très supérieure à celle du premier. Il est suggéré que dans le compartiment I se déroule l'essentiel des processus biochimiques actifs dont le sol est le siège. Tenant compte de cette hypothèse, c'est à ce compartiment que sont appliquées les données connues qui précisent, au plan quantitatif, les besoins relatifs en carbone et en azote pour que soit réalisé un équilibre entre la minéralisation et la réorganisation (immobilisation) : le rapport $\mathrm{C} / \mathrm{N}$ des substrats organiques concernés se situe généralement dans ce cas entre 25 et 30.

Le modèle présenté permet de calculer l'importance de la réorganisation de l'azote (recyclage) en fonction des apports de carbone dans le compartiment $I$. Les relations trouvées sont appliquées aux données concernant un essai de longue durée établi en sol nu, dans lequel de la paille de blé est enfouie sous différentes conditions. Tenant compte des données précédemment évoquées, le modèle permet de mettre en évidence, sur un exemple concret et pour une période suffisamment longue, la nécessité d'un recyclage de l'azote minéralisé lorsqu'une masse importante de carbone est incorporée au sol. Ainsi, dans le cas d'un enfouissement annuel de $7 \mathrm{t}$ de paille par hectare, sans apport d'azote, il est calculé que $47 \mathrm{p} .100$ de l'azote minéralisé au cours d'une année ont subi un recyclage. Un apport complémentaire d'azote minéral $(70 \mathrm{~kg} / \mathrm{ha})$ abaisse ce taux à $33 \mathrm{p} .100$ mais, par contre, augmente la quantité totale d'azote minéral immobilisé de 12 p. 100 environ. Il est évident que ces résultats, acquis sur la totalité de la couche arable, expriment en réalité la somme de comportements ponctuels : le sol peut être considéré comme un ensemble de sites de richesses fort différentes en carbone labile, en particulier après un apport extérieur nécessairement hétérogène : à un instant donné, le recyclage se développe sur le sous-ensemble des sites riches alors qu'il est inutile dans le sous-ensemble des sites pauvres. L'évaluation des taux moyens de recyclage de l'azote permet, dans le cas du sol étudié maintenu sans culture. de déterminer les taux nets annuels de minéralisation de l'azote pour les divers traitements expérimentés. Les rapports $\theta / \gamma$ des taux nets de minéralisation du carbone et de l'azote reflètent les dynamiques étroitement associées des deux éléments et, notamment, leurs relations avec les faibles variations du rapport $\mathrm{C} / \mathrm{N}$ du sol. En particulier, lorsqu'aucun apport de carbone et d'azote organiques $n$ 'est fait au sol, ce rapport $\theta / \gamma$ tend nécessairement vers 1 , valeur effectivement trouvée pour les traitements expérimentaux concernés.

\section{ABSTRACT \\ Soil mineral nitrogen, Immobilization, Organization, \\ Mineralization, \\ Bare soil, \\ Compartmental model.}

Attempt to estimate the organization and reorganization of the soil mineral nitrogen with the method of the compartment models. I. Case of an untilled soil

In this report a compartment model set up according to the unstability (or stability) criterion of the soil organic matter is proposed: a compartment I includes all the unstable organic substances living or not; a compartment II gathers the stabilized not living organic substances with a low reactivity; the last compartment represents a much more important mass than the first one.

It is assumed that the main active biochemical processes observed in the soil take place in compartment $I$. Taking this hypothesis into account we applied to this compartment the known data which precise on the quantitative level the respective demand of carbon and nitrogen in order to obtain an equilibrium between mineralization and reorganization (immobilization): the $\mathrm{C} / \mathrm{N}$ ratio of the concerned organic substrates lies generally between 25 and 30 in this case.

The model described allows to calculate the importance of nitrogen reorganization (recycling) in relation to the carbon supplies in compartment I. The data concerning a long-term experiment on an untilled soil to which straw manuring is applied under different conditions. Taking into account the data previously quoted the model makes it possible to show on a concrete example and for a sufficiently long period the necessity of a recycling of mineralized nitrogen when an important mass of carbon is introduced into the soil. Thus in the case of a yearly straw manuring of 7 tons/ha without nitrogen supply it is estimated that $47 \mathrm{p} .100$ of the mineralized nitrogen are submitted to a recycling during a year. A complementary supply of mineral nitrogen 
$(70 \mathrm{~kg} / \mathrm{ha})$ reduces this rate to $33 \mathrm{p} .100$ but increases the total amount of mineral nitrogen immobilized up to about $12 \mathrm{p} .100$. It is obvious that these results obtained on the whole topsoil express as a matter of fact the sum of punctual behaviours : the soil can be considered as a set of sites with very different resources in unstable carbon in particular after an external supply necessarily heterogeneous : at a given time the recycling occurs in the sub-unit of rich sites whereas it is useless in the sub-unit of poor sites.

The estimation of the mean rates of nitrogen recycling makes it poscible in the case of the untilled soil studied to determine the yearly net mineralization rates of nitrogen for the various treatments experimented. The ratios $\theta / \gamma$ of the net carbon and nitrogen mineralization rates indicate the closely bound dynamics of both elements and in particular their relationships with the slight variations of the soil $\mathrm{C} / \mathrm{N}$ ratio. In particular when no supply of organic carbon and nitrogen is made, this ratio $\theta / \gamma$ is necessarily close to 1 a value actually found for the experimental treatments which are concerned.

\section{INTRODUCTION}

L'azote minéral, libéré dans le sol aux dépens de l'azote organique, connaît pour l'essentiel deux destinées : l'une qui assure, au moins de façon temporaire, son immobilisation dans les structures racinaires et microbiennes, l'autre qui conduit à son élimination du sol (récoltes, lessivage, dénitrification).

Les processus de réorganisation de l'azote par les microorganismes (immobilisation), simultanés des processus de minéralisation, ont ainsi une conséquence directe sur les quantités d'azote minéral présentes à un instant donné dans le sol. Il apparaît donc intéressant de pouvoir juger des conditions dans lesquelles se produit cette réorganisation temporaire et d'en évaluer, au moins d'une façon approximative, l'importance relative. Il est bien connu que celle-ci est en rapport direct avec les quantités de carbone organique incorporées au sol et, d'une façon plus précise, avec le rapport $\mathrm{C} / \mathrm{N}$ du substrat organique apporté : à un rapport $\mathrm{C} / \mathrm{N}$ élevé, par exemple, correspond une importante réorganisation de l'azote minéral.

Nous suggérons, dans cette note, une approche du problème évoqué en proposant sa résolution par la voie de modèles compartimentaux relatifs à l'azote et au carbone. Nous appliquerons ensuite les relations théoriques ainsi élaborées aux données et résultats concernant un dispositif expérimental parcellaire de longue durée, dit «des 36 parcelles », établi en 1959 au Centre de Grignon de l'I.N.A. Paris-Grignon. Rappelons que sur ce dispositif, différents traitements sont appliqués à un sol nu maintenu sans végétation: enfouissements de paille fermentée ou non, avec ou sans apport d'azote minéral (MoREL, CHABOUIS et BOURGEOIS, 1981). Des déterminations de carbone et d'azote organiques ont été réalisées avant et après une période de 15 années, entre 1962 et 1977.

\section{MODĖLE COMPARTIMENTAL SUR LES TRANS- FERTS DE L'AZOTE DANS LE SOL}

Pour caractériser les transferts de carbone et d'azote, nous avions, dans un précédent mémoire (MOREL, 1978), établi deux modèles compartimentaux dont le critère distinctif compartimental était le caractère "vivant " ou «non vivant» de la matière organique du sol. Appliqué à l'essai que nous venons d'évoquer (dispositif des « 36 parcelles») le modèle concernant l'azote est reproduit sur la figure 1.

Le compartiment I comprend l'azote de la masse de la micropopulation, le compartiment II l'azote de l'ensemble des substances organiques non vivantes du sol dont nous connaissons l'extrême hétérogénéité tant en ce qui concerne leur composition que la stabilité de leurs différentes fractions.

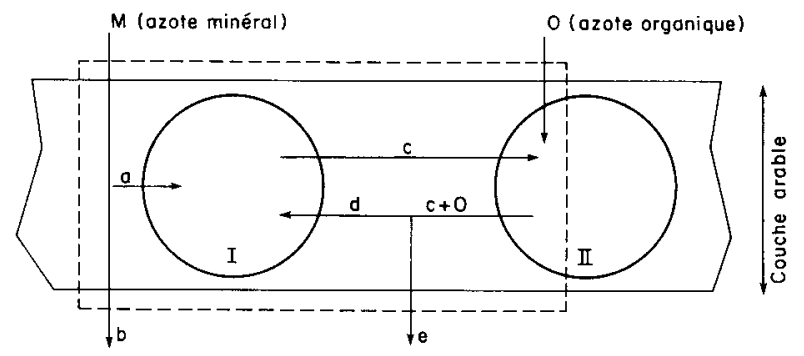

Figure 1

Modèle compartimental relatif aux transferts d'azote, appliqué à un sol sans culture. Critère distinctif: état vivant ou non vivant des substances organiques azotées.

Compartmental model of $N$ transfer applied to a non cultivated soil. Distinctive criterium : living conditions or not of the $N$ organic matter.

La quantité d'azote minéral $\mathrm{M}$, apportée au sol au cours de l'unité de temps choisie, se distribue en une partie (a) alimentant la masse microbienne et une partie (b), perdue par le sol (lessivage, dénitrification). La quantité (c) d'azote organique provient des micro-organismes morts du compartiment I; la quantité $c+O$ d'azote minéralisé se répartit entre les pertes (e) et la réorganisation par la micropopulation vivante du compartiment $I$ (d).

Nous pouvons également définir un autre modèle, proche du précédent, établi en choisissant comme critère distinctif compartimental le degré de stabilité (ou de labilité) des substances constituant la matière organique du sol, vivante ou non vivante (fig. 2).

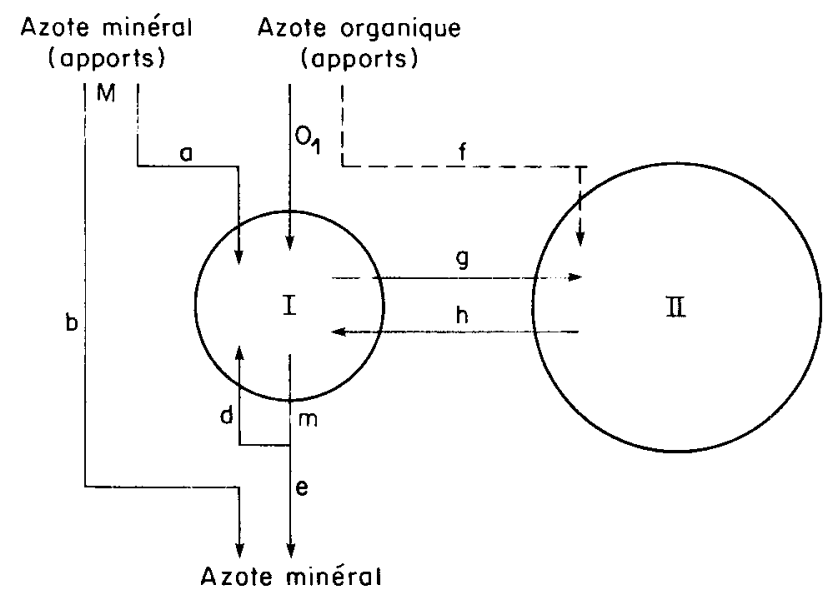

Figure 2

Modèle compartimental relatif aux transferts d'azote, appliqué à un sol sans culture. Critère distinctif: état «labile» ou "stable» des substances organiques azotées.

Compartmental model of $N$ transfer applied to a non cultivated soil. Distinctive criterion : "labile" or "stable» condition of $N$ organic matter. 
Le compartiment I est formé de l'azote des substances organiques «labiles" du sol, vivantes ou non vivantes. Le compartiment II représente l'azote des matières organiques "stables» du sol. Remarquons que ce modèle peut être considéré comme dérivé du modèle précédent en observant que le compartiment I du second modèle comprend le compartiment I du premier et la fraction «labile» du compartiment II (tireté fig. 1).

Ce critère de stabilité trouve sa justification dans les travaux qui, au cours de ces dernières années, ont permis de mieux préciser le degré de vulnérabilité des différents composés organiques du sol aux actions microbiennes. Ainsi, JENKINSON et RAYNER (1977), utilisant le ${ }^{14} \mathrm{C}$, ont pu classer 5 catégories de composés, distinguées par leur demi-vie dans le sol:

\section{1. - substances végétales} aisément décomposables

2. - substances végétales résistantes

3. - biomasse du sol

4. - matières organiques physiquement stabilisées

5. - matières organiques chimiquement stabilisées

$\begin{array}{ll}\text { demi-vie } & 0,161 \text { année } \\ \text { demi-vie } & 2,31 \quad " \\ \text { demi-vie } & 1,69 \\ \text { demi-vie } & 49,5 \quad " \\ \text { demi-vie } & 1980 \quad "\end{array}$

Deux grands groupes de substances peuvent être discernés : uñ premier groupe comprenant les trois premières catégories, dont la demi-vie est de l'ordre de quelques semaines à 2 années environ; un second groupe dont la demi-vie s'échelonne entre plusieurs décennies et plusieurs siècles. L'une des conséquences de cette distinction est que le second groupe représente dans le sol une proportion beaucoup plus grande que le premier. SAUERBECK et GONZALEZ (1976) étudiant la décomposition dans le sol de paille marquée au ${ }^{14} \mathrm{C}$, sont conduits à distinguer dans la matière organique deux pools nettement indépendants : une fraction labile dont l'origine relève des pratiques culturales, une fraction stable peu influencée par les techniques agricoles.

Citons également des recherches relatives à l'organisation structurale des complexes organo-minéraux du sol: les substances organiques fixées sous forme de couches « externes » sur le substrat minéral donneraient lieu a un turn over rapide; par contre, les fractions organiques formant des couches « internes » n'accuseraient qu'une très faible bio-dégradabilité (CHASSIN, 1980).

Dans le modèle proposé, le compartiment I, alimenté en azote par le milieu extérieur et le compartiment II, présente dès lors les activités caractéristiques suivantes:

- minéralisation rapide des substances organiques « non vivantes";

- édification dans sa masse des substances organiques stables qui alimentent le compartiment II ;

- renouvellement permanent de la biomasse grâce à la présence simultanée d'azote, de carbone et des autres éléments nutritifs.

Nous postulons ainsi le fait que dans le compartiment I se déroule avec intensité l'essentiel des processus fondamentaux de l'évolution de la matière organique dans le sol et, en particulier, la minéralisation et l'humification ; cela implique comme corollaire que, dans ce compartiment, se développe une part très largement prépondérante des transferts énergétiques de caractère biochimique dont le sol est le siège.

L'azote minéralisé peut connaître deux destinées : l'une conduit à son élimination du sol : lessivage, dénitrification... (e) ; l'autre l'appelle à une réorganisation par la micropopulation, constituant ainsi un "recyclage" au niveau même du compartiment I (d). Il ne fait aucun doute que ce recyclage peut être très rapide; de plus, une molécule d'azote, considérée individuellement, est susceptible d'être recyclée de nombreuses fois avant de se trouver éliminée définitivement du sol (e).

Le compartiment II rassemble, quant à lui, la fraction quantitativement la plus importante de l'azote organique du sol, de réactivité très faible et de ce fait, la plus stable. Nous considérons que ce compartiment est alimenté de façon permanente par l'effet des processus biochimiques qui se produisent dans le compartiment I ; toutefois, il peut éventuellement recevoir de façon directe du milieu extérieur des substances organiques stabilisées par des actions préalables et étrangères au sol (substances préhumifiées).

Enfin, nous formons également l'hypothèse que la dégradation des substances azotées stables du compartiment II conduit à la genèse de substances organiques labiles qui alimentent le compartiment I ; en d'autres termes, nous supposons qu'il n'y a pas production directe de composés azotés minéraux $\left(\mathrm{NH}_{4}^{+}\right)$à partir du compartiment II.

Notons aussi qu'entre les compartiments I et II se dessine un cycle de l'azote $(g+h)$ dont la période moyenne est beaucoup plus longue que la période du cycle observé au niveau du seul compartiment I (d).

\section{MODÉLE COMPARTIMENTAL SUR LES TRANS- FERTS DE CARBONE DANS LE SOL}

Le critère de stabilité (ou de labilité) des substances organiques conduit à l'élaboration d'un modèle compartimental simple (fig. 3). Cette simplicité tient au fait que l'alimentation du compartiment I aux dépens de carbone « minéral » est d'une importance qualitative minime (microorganismes autotrophes) et nous l'avons négligée.

De même que pour l'azote, le modèle comprend deux compartiments dont les traits essentiels ont été définis. Le compartiment I est alimenté en carbone par les apports extérieurs ainsi que par les substances labiles issues de la dégradation des composés organiques du compartiment II (flèches 1 et 5).

Le dioxyde de carbone, libéré lors des processus de minéralisation, est dégagé dans l'atmosphère du sol, ne donnant lieu qu'à un recyclage d'une faible importance relative et dont nous ne tiendrons pas compte. Remarquons, par contre, qu'un recyclage notable met en jeu des substances organiques issues de la micropopulation morte et qui sont réincorporées dans la biomasse du fait du caractère hétérotrophe de celle-ci (flèche 6).

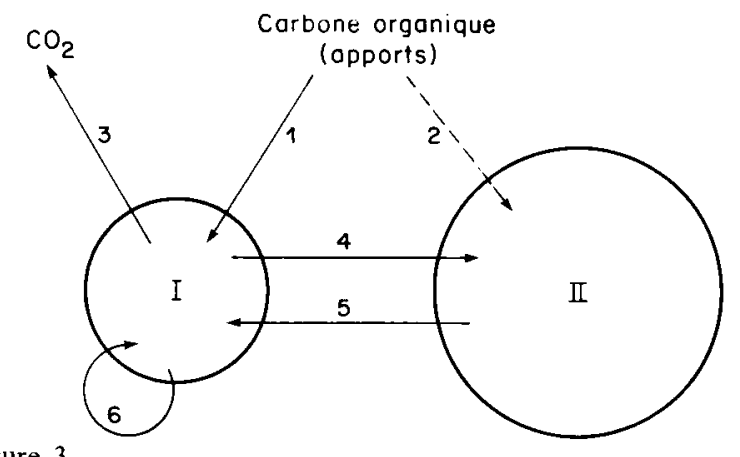

Figure 3

Modèle compartimental relatif aux transferts de carbone, appliqué à un sol sans culture. Critère distinctif : état "labile» ou "stable" des substances organiques.

Compartmental model of $C$ transfer applied to a non cultivated soil. Distinctive criterium : "labile " or "stable" condition of the organic matters. 
Le compartiment II reçoit du compartiment I une masse carbonée formée de substances en cours de stabilisation (flèche 4) et s'enrichit éventuellement d'un apport extérieur (flèche 2). Ainsi que nous l'avons proposé pour l'azote, nous supposerons que la forme minérale du carbone $\left(\mathrm{CO}_{2}\right)$ n'est acquise qu'aux dépens du compartiment I.

\section{RELATIONS ENTRE LE CARBONE ET L'AZOTE}

Les évolutions du carbone et de l'azote ne sont pas indépendantes l'une de l'autre. Les quantités de carbone nécessaires au déroulement permanent du métabolisme cellulaire s'ajoutent aux besoins qui associent carbone et azote dans l'édification des structures de la cellule vivante (assimilation). Si la quantité d'azote apportée par le substrat organique mis en cuvre est trop faible par rapport à la quantité de carbone, l'azote libéré par les processus de minéralisation est aussitôt réintégré dans les masses microbiennes tandis qu'un complément est sollicité de la part du milieu extérieur. De l'azote minéral apparaît au contraire dans le milieu lorsque le substrat organique est riche en cet élément.

Un ensemble de travaux réalisés sur cette question conduit à admettre que pour un matériel végétal contenant 40 p. 100 de carbone, un taux d'azote de l'ordre de 1,5 p. 100 représente le niveau pour lequel il existe un équilibre entre les deux processus : minéralisation et réorganisation (immobilisation). Ce niveau s'établit donc pour un rapport $\mathrm{C} / \mathrm{N}$ du substrat avoisinant 27 (valeur que nous utiliserons pour la simplicité des calculs) : au-dessous de ce rapport, le substrat libère de l'azote minéral, au-dessus, celui-ci n’apparaît pas dans le milieu : il est réorganisé ou « recyclé » (HARMSEN \& VAN SCHREVEN, 1955 ; BARTHOLOMEW, 1965 ; ALLISON, 1966 ; BLACK, 1968 ; AlEXANDER, 1977 ; SCHNITZER \& KHAN, 1978).

Nous référant alors au modèle compartimental proposé, nous suggérons d'utiliser ces données quantitatives en les appliquant aux processus de minéralisation et de réorganisation qui se déroulent dans le compartiment I caractérisé, à l'inverse du compartiment II, par une activité microbienne très intense.

\section{RELATIONS QUANTITATIVES}

Nous nous placerons dans le cas où, les apports extérieurs demeurant constants, les taux de carbone et d'azote organiques du sol diminuent au cours de l'unité de temps choisie ; la démarche développée ci-après est également valable pour un sol dont les taux de carbone et d'azote augmentent.

Nous établirons notre raisonnement sur l'étude des transferts de carbone et d'azote qui, au cours de l'unité de temps, affectent le compartiment I :

- apports extérieurs organiques et minéraux (fig. 2),

- apports organiques en provenance du compartiment II,

- sorties sous forme de composés minéraux.

Notons ici une remarque : les pertes du sol proviennent pour une part du compartiment II (apports organiques au compartiment I), pour une autre fraction du compartiment I. Cependant, quelle que soit leur origine compartimentale, ces pertes participent au même titre avant leur minéralisation à l'ensemble des activités réactionnelles du compartiment I, activités qui, en définitive, conduisent l'azote et le carbone à des formes minérales.

Soit alors A la quantité d'azote que le compartiment I doit recevoir pour que s'établisse un équilibre entre les deux processus simultanés de minéralisation et de réorganisation; A est obtenue en divisant par 27 la quantité de carbone «labile " mise en cuvre dans le compartiment I, formée de deux termes:

- le premier représente l'apport $\mathrm{C}_{1}$ de carbone au sol et que nous supposerons en totalité dirigé vers le compartiment I,

- le second exprime la diminution $\mathrm{C}_{2}$ de la quantité de carbone organique du sol (pertes); rappelons que nous avons postulé que la forme minérale du carbone $\left(\mathrm{CO}_{2}\right)$ n'est issue que du seul compartiment I.

\section{Soit encore :}

M la quantité d'azote minéral apportée au sol (fig. 2),

O la quantité d'azote organique mise en jeu dans le compartiment I au cours de l'unité de temps et composée, d'une façon homologue de celle relative au carbone, de deux termes:

- la masse $O_{1}$ incorporée au sol sous forme organique, d'une part,

- la masse $\mathrm{O}_{2}$ qui représente la perte d'azote organique du sol, d'autre part ; ainsi que nous l'avons indiqué, cette quantité perdue, issue des deux compartiments, participe dans sa totalité aux activités biochimiques du compartiment I.

L'ensemble de données ainsi défini permet d'établir plusieurs relations qui conditionnent les transferts d'azote au niveau du compartiment $I$.

$$
\begin{gathered}
M=a+b \\
A-O=a+d
\end{gathered}
$$

Ainsi que nous l'avons exprimé, si A excède $O$, un apport d'azote minéral devient nécessaire, qui relève de deux origines possibles : l'une est un prélèvement (a) de l'azote minéral apporté au sol ; l'autre (d), représente la fraction réorganisée de l'azote minéralisé dans le compartiment I. En d'autres termes, la différence $(\mathrm{A}-\mathrm{O})$ est comblée par les quantités d'azote (a) et (d), ce qui s'écrit bien $\mathrm{A}-\mathrm{O}=\mathrm{a}+\mathrm{d}$.

Toutefois, l'importance relative des prélèvements (a) et (d) ne présente vraisemblablement pas un caractère aléatoire et nous suggérons d'appliquer l'hypothèse suivante, déjà proposée dans une communication antérieure (MOREL, 1978): pour assurer son alimentation azotée, le microorganisme ne fait aucune distinction entre l'azote minéral apporté au sol (engrais) et l'azote issu de la minéralisation des composés organiques du compartiment I. Si m désigne la quantité d'azote minéralisée au cours de l'unité de temps, cette hypothèse s'exprime dans les relations:

$$
\begin{aligned}
& \frac{\mathrm{a}}{\mathrm{M}}=\frac{\mathrm{d}}{\mathrm{m}} \\
& \frac{\mathrm{b}}{\mathrm{M}}=\frac{\mathrm{e}}{\mathrm{m}} .
\end{aligned}
$$

Enfin, une dernière relation peut être déduite du bilan des transferts d'azote dans le compartiment I au cours de l'unité de temps choisie. 
Soit X la masse d'azote organique du compartiment au début de cette unité de temps et $q$ sa diminution au cours de cette même période. Au terme de celle-ci, la quantité d'azote restante est alors égale à $(X-q)$.

Le bilan de l'azote dans le compartiment I s'écrit alors :

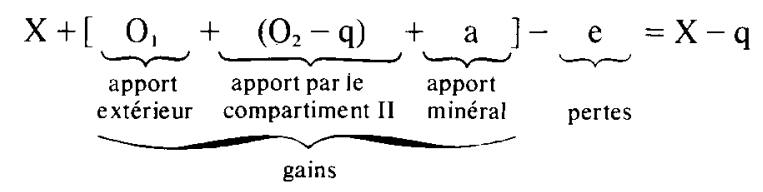

d'où

$$
\mathrm{e}=\mathrm{O}_{1}+\mathrm{O}_{2}+\mathrm{a} \text { et } \mathrm{e}=\mathrm{O}+\mathrm{a} .
$$

La résolution du système représenté par les cinq équations précédentes conduit aux valeurs de $\mathrm{a}, \mathrm{b}, \mathrm{d}$, e et $\mathrm{m}$.

$$
\begin{gathered}
a=\frac{M(A-O)}{M+A} \quad d=\frac{A(A-O)}{M+A} \\
b=\frac{M(M+O)}{M+A} \quad e=\frac{A(M+O)}{M+A} \\
m=A
\end{gathered}
$$

Les rapports $\frac{\mathrm{a}}{\mathrm{b}}$ et $\frac{\mathrm{d}}{\mathrm{e}}$, égaux, ont pour expression $\frac{\mathrm{A}-\mathrm{O}}{\mathrm{M}+\mathrm{O}}$.

La réorganisation de l'azote peut être définie par un «taux de réorganisation» ou "taux de recyclage », qui a pour valeur :

$$
\frac{\mathrm{d}}{\mathrm{m}}=\frac{\mathrm{a}}{\mathrm{M}}=\frac{\mathrm{A}-\mathrm{O}}{\mathrm{M}+\mathrm{A}}
$$

Soulignons que ce taux de recyclage est identique au taux d'organisation de la quantité d'azote minéral $M$. Notons aussi que ce taux dépend, entre autres, de la masse d'azote minéral $M$ apportée au sol (relation 6) ; généralement et pour des déficits $(\mathrm{A}-\mathrm{O})$ du même ordre de grandeur, il augmente lorsque la quantité d'azote minéral incorporée au sol s'abaisse.

Notons aussi que la masse d'azote organique minéralisée $(m=A)$ excède de la quantité $(A-O)$ les apports $O$ d'azote au compartiment $I$.

La réorganisation cesse si $\mathrm{O} \geqslant \mathrm{A}$ (taux de recyclage nul). Dans ce cas, les 5 relations $1,2,3,4$ et 5 se réduisent à deux :

$$
\begin{aligned}
\mathrm{M} & =\mathrm{b} \\
\mathrm{e} & =0 .
\end{aligned}
$$

L'azote minéral apporté, demeurant libre dans le sol, n'intervient plus dans le système compartimental. La quantité $m$ d'azote minéralisé est égale aux apports au compartiment I : $\mathrm{m}=\mathrm{O}$

\section{APPLICATIONS}

\subsection{Organisation et réorganisation de l'azote}

Rapportons ces résultats théoriques aux données relatives au dispositif expérimental des « 36 parcelles», dont les différents traitements du sol, maintenu sans végétation, sont les suivants :

1. - sans azote minéral, sans enfouissement de paille
2. - sans azote minéral, avec enfouissement de paille

3. - sans azote minéral, avec enfouissement de paille préhumifiée

4. - avec azote minéral, sans enfouissement de paille

5. - avec azote minéral, avec enfouissement de paille

6. - avec enfouissement de paille préhumifiée en présence d'azote minéral.

Les quantités de paille enfouies sont de $7000 \mathrm{~kg}$ par ha et par an ; l'azote minéral est incorporé au sol sous forme de nitrate d'ammonium à la dose de $70 \mathrm{~kg}$ par ha et par an. La préhumification de la paille a pour conséquence une diminution importante $(60$ p. 100 environ) des quantités de carbone dans la paille fermentée. Les quantités d'azote, quant à elles, demeurent voisines dans les cas de préhumification avec ou sans apport d'azote (tabl. 1). Ce fait, important à noter, tient à ce que la paille fermentée sans azote fixe de l'azote de l'air tandis que la paille traitée en présence d'azote perd une fraction importante de cet élément au cours de la fermentation (MOREL, ChaBouIs, Bourgeois, 1981).

Dans tous les cas, les teneurs en azote et carbone organiques se sont abaissées d'une façon nettement plus marquée en l'absence d'apports extérieurs organiques.

Rapportés à une année, les données expérimentales et les résultats calculés sont reproduits sur le tableau 1. Les quantités de carbone et d'azote organiques représentent, pour chacun des deux éléments, la somme des apports extérieurs et des pertes propres du sol.

Pour les traitements $1,3,4$ et 6 , les quantités $O$ d'azote organique mises en œuvre sont supérieures aux quantités $\mathrm{A}$ caractérisant le niveau d'équilibre minéralisation-réorganisation. Le recyclage de l'azote minéralisé n'est pas nécessaire, non plus que la participation (a) de l'azote minéral apporté à l'activité biochimique du compartiment $I$.

Par contre, dans les traitements 2 et 5 , comportant un apport important de paille non préhumifiée, les quantités de carbone mises en jeu imposent une réorganisation de l'azote minéralisé (d) et une intervention de l'azote minéral apporté au sol (a). Les taux de recyclage sont respectivement de 47 et 33 p. 100 . Notons que la quantité totale d'azote minéral «immobilisée » $(\mathrm{a}+\mathrm{d})$ est légèrement augmentée (12 p. 100 environ) par l'apport d'engrais azoté. Mais, inversement, le taux de recyclage apparaît dans ce cas abaissé de façon sensible (action du facteur $M$ ).

\section{Remarques}

1) Les valeurs calculées expriment l'ampleur de la réorganisation de l'azote pour une période suffisamment longue, une année par exemple ; ainsi, dans le traitement $\mathrm{n}^{\circ} 2$ (sans azote, avec paille), 46,6 p. 100 de l'azote minéralisé ont subi un recyclage; un taux identique affecte l'azote minéral utilisé directement par la micropopulation du sol (a, fig. 2).

Toutefois, ces valeurs ne demeurent pas constantes dans le temps ; ainsi après un enfouissement de paille, le taux de recyclage, considéré pour l'ensemble du sol, est élevé par le fait d'un apport important de carbone au sol. Il devient nul dès que le rapport $\mathrm{C} / \mathrm{N}$ des substances organiques en évolution dans le compartiment I s'est suffisamment abaissé

2) Dans le cas où un apport important de carbone organique impose un recyclage de l'azote, ce dernier s'établit ou ne se réalise pas selon le site du sol considéré ; en effet, en raison de l'hétérogénéité inévitable de la répartition du carbone «labile», tel site peut connaître temporairement une réorganisation importante de l'azote alors que le recyclage se révèle inutile en un point voisin. 


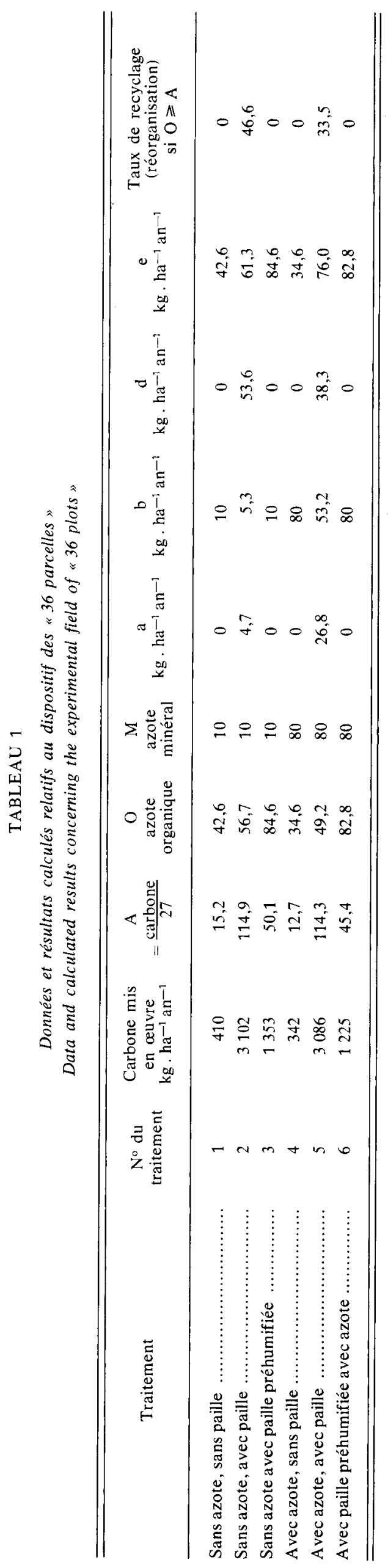


Précisons que les sites considérés peuvent être très petits, à l'échelle dimensionnelle des micro-organismes, chacun d'eux présentant à un instant donné son propre taux de recyclage.

Il résulte de cette remarque que, considéré pour l'ensemble du compartiment I, le taux de recyclage n'atteint vraisemblablement jamais l'unité, même après l'apport au sol d'un substrat à rapport $\mathrm{C} / \mathrm{N}$ élevé.

Inversement, il n'apparaît pas impossible qu'après l'incorporation d'une substance relativement pauvre en carbone, il subsiste pendant un certain temps des sites dont le rapport $\mathrm{C} / \mathrm{N}$ implique un recyclage de l'azote : le taux moyen de réorganisation n'est alors pas nul pour la période considérée.

3) Dans les applications présentées, le rapport $\mathrm{C} / \mathrm{N}$ correspondant à l'équilibre minéralisation-réorganisation a été choisi égal à 27 . Cette valeur n'exprime en réalité qu'un ordre de grandeur sans aucun doute variable selon la nature et les proportions relatives des différentes espèces microbiennes présentes à un instant donné dans le site de sol concerné. Il n'est cependant pas impossible que, pour l'ensemble de tous les sites de la couche arable, ces variations demeurent entre des limites relativement étroites, correspondant aux conditions pédoclimatiques et culturales de la parcelle considérée.

4) La présence d'azote minéral sur un site du sol (provenant de la minéralisation ou des apports extérieurs) est directement fonction de l'existence ou de la non existence de recyclage sur ce site. Pour l'ensemble de la couche arable, la quantité totale d'azote minéral, à un instant donné, est la somme des masses ponctuelles de cette forme d'azote présentes à cet instant sur tous les sites du sol.

\subsection{Taux nets annuels de minéralisation de l'azote et du carbone}

Ces taux peuvent être calculés par l'application d'une relation générale proposée dans un précédent mémoire, concernant un sol qui n'a pas atteint son équilibre organique (MOREL, 1978).

Le rapport $\omega$, figurant dans cette relation, exprime la proportion de l'azote minéralisé qui est réorganisée, ou bien la fraction de l'azote minéral apporté organisée dans les racines et la micropopulation. Dans le cas présent (sol maintenu sans végétation), la réorganisation ne concerne que la micropopulation du sol. Nous pouvons ainsi assimiler $\omega$ à la grandeur définie par les rapports $\mathrm{d} / \mathrm{m}=(\mathrm{A}-\mathrm{O}) /(\mathrm{M}+\mathrm{A})$ et que nous avons appelés « taux de recyclage " ou " taux de réorganisation » (tabl. 1). Il est alors possible de calculer sans ambiguité les taux nets $\gamma$ de minéralisation de l'azote organique du sol. Le tableau 2 rapporte les résultats obtenus que nous avons complétés par les taux nets de minéralisation $\theta$ du carbone organique du sol; ces derniers ont été calculés par l'application d'une relation homologue de celle utilisée pour la détermination de $\gamma$ (MOREL, 1978).

Notons sur ce tableau que la préhumification de la paille a pour effet une diminution très marquée de $\theta$. Cela tient au fait que 60 p. 100 environ du carbone initial de la paille sont perdus lors de la préhumification (MOREL, CHABOUIS et BOURGEOIS, 1981); aussi, l'importance de cette perte se retrouve-t-elle dans le rapport des taux nets de minéralisation du carbone. L'apport d'azote, par contre, n'a que peu d'incidence sur les valeurs de $\theta$.

Ce tableau souligne également le fait que la valeur de $\theta / \gamma$ apparaît directement liée à la faible variation du rapport $\mathrm{C} / \mathrm{N}$ malgré la diversité des traitements ( $\mathrm{C} / \mathrm{N}$ voisin de 9) : aussi, pour assurer l'établissement dans le sol d'un rapport $\mathrm{C} / \mathrm{N}$ constant, l'incorporation d'un substrat organique à rapport $\mathrm{C} / \mathrm{N}$ élevé induit-elle nécessairement un rapport $\theta / \gamma$ également élevé. Inversement, lorsque pendant une longue période, il n'est effectué aucun apport de carbone et d'azote organiques au sol, il doit en résulter des valeurs semblables des taux nets de minéralisation $\theta$ et $\gamma$, condition nécessaire à la réalisation d'un rapport $\mathrm{C} / \mathrm{N}$ constant : le rapport $\theta / \gamma$ tend alors vers 1 , ce qui est observé dans les traitements 1 et 4 bien que le sol n'ait pas encore atteint son état d'équilibre organique.

\section{CONCLUSIONS}

Trois traits essentiels caractérisant la matière organique du sol ont suggéré une étude quantitative de son évolution par référence à un modèle bicompartimental.

a) la division de l'ensemble des substances organiques, vivantes ou non vivantes, en deux groupes distincts par leur persistance plus ou moins longue dans le sol;

b) la nature hétérotrophe de la plus grande partie de la micropopulation du sol, celle-ci ne pouvant utiliser le dioxyde de carbone, terme final minéral de l'évolution biochimique du carbone des composés organiques. Le $\mathrm{CO}_{2}$ produit gazeux, s'élimine du milieu réactionnel dès sa formation; par contre, les composés minéraux azotés, présents sous forme de solutions ioniques, demeurent au moins temporairement sur leurs sites de formation et, entre

TABLEAU 2

Taux nets de minéralisation du carbone organique $(\theta)$ et de l'azote organique du sol ( $\gamma)$ (taux calculés) Calculated constants of the soil organic carbon $(\theta)$ and soil organic nitrogen ( $\gamma$ ) decomposition per annum

\begin{tabular}{|c|c|c|c|c|c|c|}
\hline Traitements & $\begin{array}{l}n^{\circ} \mathrm{du} \\
\text { traitement }\end{array}$ & $\theta \times 100$ & $\gamma \times 100$ & $\theta / \gamma$ & $\alpha$ & $\begin{array}{c}\text { Rapport } \mathrm{C} / \mathrm{N} \\
\text { des apports } \\
\text { organiques }\end{array}$ \\
\hline Sans azote, sans paille...$\ldots \ldots \ldots \ldots \ldots \ldots \ldots$ & 1 & 1,22 & 1,13 & 1,07 & 1,13 & \\
\hline Sans azote, avec paille ......................... & 2 & 8,34 & 1,53 & 5,45 & 2,87 & 85,3 \\
\hline Sans azote, avec paille préhumifiée $\ldots . . . \ldots$. & 3 & 3,65 & 2,11 & 1,73 & 2,11 & 21,6 \\
\hline 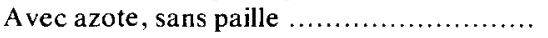 & 4 & 0,97 & 0,89 & 1,09 & 0,89 & \\
\hline Avec azote, avec paille...$\ldots \ldots \ldots \ldots \ldots \ldots$ & 5 & 8,23 & 1,86 & 4,43 & 2,80 & 85,3 \\
\hline Avec paille préhumifiée avec azote .......... & 6 & 3,21 & 2,03 & 1,58 & 2,03 & 16,9 \\
\hline
\end{tabular}

$\alpha=$ taux réel (brut) de minéralisation calculé en fonction de $\alpha$ et $\omega$ (MORE1., 1978). 
autres destinées, peuvent donner lieu à de nombreux recyclages de l'azote. Ces traits distinctifs des composés minéraux des deux éléments apparaissent ainsi comme des propriétés essentielles dans l'orientation des dynamiques - espectives du carbone et de l'azote dans le sol.

c) le caractère énergétique qui marque chaque étape de l'activité biochimique du sol. L'organisation structurale du micro-organisme se réalise sur un rapport $\mathrm{C} / \mathrm{N}$ de l'ordre de 10 ; mais l'édification et le maintien des structures vivantes sollicitent une dépense de composés carbonés très supérieure aux nécessités structurales proprement dites. Pour un fonctionnement microbien normal, un équilibre s'établit entre les besoins en carbone et en azote, équilibre qui, pour l'ensemble de la micropopulation d'un sol donné, se situe à un niveau sans aucun doute fonction des caractéristiques pédoclimatiques et culturales de la parcelle concernée. Il apparaîtrait souhaitable, sous différentes conditions, de pouvoir évaluer de façon plus précise ce niveau afin de mieux appréhender l'aspect quantitatif de l'évolution des substances organiques dans le sol.

Reç le 5 novembre 1979. Accepté le 10 octobre 1980.

\section{REMERCIEMENTS}

Nous désirons exprimer nos vifs remerciements à MM. C. JUSTE et J. C. Rémy (respectivement Stations d'agronomie I.N.R.A. de Bordeaux et de Laon) avec lesquels nous avons eu de fructueux échanges de vues et qui ont bien voulu nous faire part de leurs observations sur ce mémoire.

\section{REFERENCES BIBLIOGRAPHIQUES}

Alexander M., 1977. Introduction to soil microbiology, Second Edition, John Wiley and Sons, $467 \mathrm{p}$.

Allison F. E., 1966. The fate of nitrogen applied to soils. $A d l$. Agron., 18, 219-258.

Bartholomew W. V., 1965. Mineralization and immobilization of nitrogen in the decomposition of plant and animal residues in Soil Nitrogen, American Society of Agronomy, Madison, Wisconsin. 285-306.

Black C. A., 1968. Soil-Plant relationships, Second Edition. John Wiley and Sons, $792 \mathrm{p}$.

Chassin P., 1980. Hypothèse sur la structure du complexe argilohumique. Conséquences sur les processus d'humification. (A paraître dans Sci. Sol).

Jenkinson D. S., Rayner J. H., 1977. The turnover of soil organic matter in some of the Rothamsted classical experiments. Soil Sci. . 123 (5). 298-305.
Harmsen G. W., Van Schreven D. A., 1955. Mineralization of organic nitrogen in soil. Adv. Agron., 7, 300-398.

Morel R., 1978. Quelques aspects nouveaux de la dynamique du carbone et de l'azote dans le sol. Ann. Agron., 29 (4). 357-379.

Morel R., Chabouis Catherine, Bourgeois S., 1981. Evolution des taux d’azote et de carbone organiques dans un sol nu après enfouissement de paille sous différentes conditions. Agronomie 1 (1), $7-18$

Sauerbeck D. R., Gonzalez M. A., 1976. Field decomposition of carbon 14 labelled plant residues in various soils of the Federal Republic of Germany and Costa Rica. Soil Organic Matter Studies. Proceedings of a symposium, Braunschweig 1976, vol. I, 159-170.

Schnitzer M., Khan S. U., 1978. Soil organic Matter. Serie : Developments in soil science $(8), 319$ p. Elsevier. 